\title{
Developments in the measurement of spectral momentum densities with $(\mathrm{e}, 2 \mathrm{e})$ spectrometers
}

\author{
Maarten Vos, Erich Weigold* \\ Atomic and Molecular Physics Laboratories, Research School of Physical Sciences and Engineering, Institute of Advanced Studies, \\ Australian National University, Canberra ACT 0200, Australia
}

Received 8 March 2000; accepted 13 April 2000

\begin{abstract}
The introduction of multiparameter high energy electron momentum spectroscopy (EMS) spectrometers, with high energy and momentum resolution and coincidence count rates, has made it possible to obtain detailed information on the electronic structure of condensed matter and surfaces. In particular it gives direct information on the spectral-momentum density of the material under study, which may be a single crystal, polycrystalline, or amorphous. The first such spectrometer, the Flinders University spectrometer, uses asymmetric kinematics in the measurement of the energies and momenta of the two outgoing electrons in the $(\mathrm{e}, 2 \mathrm{e})$ collision. The new ANU spectrometer uses symmetric kinematics and much higher energies. It is therefore less affected by deleterious multiple-scattering events. On the other hand the Flinders spectrometer is surface sensitive, whereas the ANU spectrometer is more bulk sensitive. Some aspects of EMS measurements of condensed-matter specimens and the performance of the two spectrometers are discussed. (C) 2000 Elsevier Science B.V. All rights reserved.
\end{abstract}

Keywords: EMS; Spectral-momentum density; Scattering kinematics

\section{Introduction}

The energy-momentum densities of electrons in atoms, molecules and solids can be directly measured using kinematically complete ionisation reactions initiated by electron beam, i.e. (e,2e) collisions (Weigold and McCarthy [1]). At high enough energies and momentum transfer the (e,2e) differential cross section, as a function of the difference between initial and total final electron momenta and energies, is proportional to the electron energy-

\footnotetext{
*Corresponding author. Tel.: +61-2-6249-2476; fax: +61-26249-5457.

E-mail address: director.rspse@anu.edu.au (E. Weigold).
}

momentum density of the target specimen. The energy

$\epsilon_{i}=E_{0}-E_{\mathrm{s}}-E_{\mathrm{f}}$,

and momentum

$\mathbf{p}=\mathbf{k}_{0}-\mathbf{k}_{\mathrm{s}}-\mathbf{k}_{\mathrm{f}}$,

correspond to the binding energy $\epsilon_{i}$ and momentum $\mathbf{q}=-\mathbf{p}$ of the struck electron. The subscripts $0, \mathrm{~s}$, and $\mathrm{f}$ label the energies $E$ and momenta $\mathbf{k}$ of the incident, and slow and fast outgoing electrons, respectively. The momentum transferred by the fast (often called the 'scattered') electron

$$
\mathbf{K}=\mathbf{k}_{0}-\mathbf{k}_{\mathrm{f}}
$$

must also be large to ensure a clean knockout of the 
electron (the binary-encounter approximation). The kinematic variables that are measured for electron momentum spectroscopy (EMS) are normally restricted to be near the Bethe ridge where

$K=k_{\mathrm{s}}$.

The geometry that ensures $K$ and $E_{\mathrm{s}}$ are maximised for any $E_{0}$ is the symmetric geometry, in which $E_{\mathrm{s}}=E_{\mathrm{f}}$ and $\theta_{\mathrm{s}}=\theta_{\mathrm{f}}=\theta$, with the most frequently used arrangement being non-coplanar symmetric kinematics in which $\theta$ is fixed near $45^{\circ}$ and the relative azimuthal angle

$\phi=\phi_{\mathrm{f}}-\phi_{\mathrm{s}}+\pi$

is varied to vary the recoil momentum p (Eq. (2)) (McCarthy and Weigold [2]) The (e,2e) differential cross section for ionisation is [3]

$\frac{\mathrm{d}^{5} \sigma}{\mathrm{d} \Omega_{\mathrm{f}} \mathrm{d} \Omega_{\mathrm{s}} \mathrm{d} E_{\mathrm{f}}}=(2 \pi)^{4} \frac{k_{\mathrm{f}} k_{\mathrm{s}}}{k_{0}} \sum_{\mathrm{av}}\left|\left\langle\mathbf{k}_{\mathrm{f}} \mathbf{k}_{\mathrm{s}} I|T| G \mathbf{k}_{0}\right\rangle\right|^{2}$.

$G$ and $I$ are, respectively, the initial $N$-electron target and residual $N-1$ electron 'ion' states and $\Sigma_{\mathrm{a} v}$ denotes a sum for final states and average for initial states that are not resolved in the experiment and can be considered as degenerates. The operator $T$ governs the transition from the entrance channel to exit channel. In the binary-encounter approximation $T$ depends on the coordinates of only the projectile and ejected electrons. The structure amplitude in Eq. (6) then factorises in the impulse approximation, and the differential cross section is given as the product of an electron-electron collision factor $f_{\mathrm{ee}}$ and a structure factor

$\frac{\mathrm{d}^{5} \sigma}{\mathrm{d} \Omega_{\mathrm{f}} \mathrm{d} \Omega_{\mathrm{s}} \mathrm{d} E_{\mathrm{f}}}=(2 \pi)^{4} \frac{k_{\mathrm{f}} k_{\mathrm{s}}}{k_{0}} f_{\mathrm{ee}} \sum_{\mathrm{av}} A(\mathbf{q}, \boldsymbol{\epsilon})$,

where the energy-momentum spectral function is given as the imaginary part of the one-particle Green's function

$A(\mathbf{q}, \epsilon)=|\langle\mathbf{q} i \mid 0\rangle|^{2} \delta\left(\epsilon-\epsilon_{i}\right)=\operatorname{Im} \frac{1}{\pi} G(\mathbf{q}, \epsilon)$.

Here the notation 0 and $i$ are used for the initial and final electronic states. In the Born-Oppenheimer approximation the vibrational and rotational degrees of freedom have been separated out, so that for a molecular target $\Sigma_{\mathrm{a} v}$ denotes that the spectral function is spherically averaged and vibrationally aver- aged [1,2]. The latter is well approximated by calculating the spectral function at the target state equilibrium nuclear positions [4]. A crystal may be considered as a large molecule that cannot rotate, vibrational excitations (phonons) are again not resolved.

The structure amplitude $\langle\mathbf{q} i \mid 0\rangle$ in Eq. (8) is a one-electron function as it involves integration over the coordinates of the $(N-1)$ electrons common to the initial and final states. In atomic and molecular physics this function is called the quasiparticle or Dyson orbital $\phi_{\alpha}\left(\mathbf{q}, \epsilon_{i}\right)$

$\langle\mathbf{q} i \mid 0\rangle=\left(S_{i}^{\alpha}\right)^{1 / 2} \phi_{\alpha}\left(\mathbf{q}, \epsilon_{i}\right)$,

where $S_{i}^{\alpha}$ is the spectroscopic factor or pole strength giving the probability that the ion state $i$ consists of the target state $|0\rangle$ with a hole in the orbital $\phi_{\alpha}$.

The form of the electron-electron collision factor $f_{\text {ee }}$ is obtained from the Ford [5] half-on-shell Coulomb T-matrix element (to allow for the finite binding energy):

$$
\begin{aligned}
f_{\mathrm{ee}} & =\frac{1}{\left(2 \pi^{2}\right)^{2}} \frac{2 \pi \eta}{e^{2 \pi \eta}-1}\left[\frac{1}{\left|\mathbf{k}_{0}-\mathbf{k}_{\mathrm{f}}\right|^{4}}+\frac{1}{\left|\mathbf{k}_{0}-\mathbf{k}_{\mathrm{s}}\right|^{4}}\right. \\
& \left.-\frac{1}{\left|\mathbf{k}_{0}-\mathbf{k}_{\mathrm{f}}\right|^{2}} \frac{1}{\left|\mathbf{k}_{0}-\mathbf{k}_{\mathrm{s}}\right|^{2}} \cos \left(\eta \ln \frac{\left|\mathbf{k}_{0}-\mathbf{k}_{\mathrm{s}}\right|^{2}}{\left|\mathbf{k}_{0}-\mathbf{k}_{\mathrm{f}}\right|^{2}}\right)\right], \\
\eta & =1 /\left|\mathbf{k}_{\mathrm{f}}-\mathbf{k}_{\mathrm{s}}\right| .
\end{aligned}
$$

This expression reduces to the Born Approximation if the Coulomb parameter $\eta$ is negligible. The dependence of the cross section on $K^{-4}$ can be seen as arising from the electron-electron collision factor, and so large $K$ corresponds to small cross sections. In non-coplanar kinematics, all the terms in Eq. (10) are constant except for very small variations in $\eta$, which are generally negligible. Thus, the $(\mathrm{e}, 2 \mathrm{e})$ cross section (Eq. (7)) as a function of recoil momentum $\mathbf{q}$ and binding energy $\epsilon$ is directly proportional to the spectral density $A(\mathbf{q}, \epsilon)$. This contains much more information than just the band position in energy, ie. dispersion. The magnitude of $A(\mathbf{q}, \epsilon)$ is the probability of the electron (or more generally the quasiparticle) having momentum $\mathbf{q}$ and energy $\epsilon$. The width of the main quasi-particle peak in $A(\mathbf{q}, \epsilon)$ gives the quasi-particle lifetime at momentum q. Due to electron correlations additional structures or satellites may be contained in $A(\mathbf{q}, \epsilon)$. All this can be measured 
by EMS, but the performance of the technique depends on the precision with which the experimental results are obtained.

Important parameters to be considered in any EMS measurement are incident energy $E_{0}$, momentum transfer $K$, coincidence count rate and the corresponding signal to background ratios, energy and momentum resolution, and elastic and inelastic collisions by the incident and emitted electrons (known as multiple scattering effects).

The energy resolution in (e,2e) measurements on solids has improved from around $150 \mathrm{eV}$ in the 30 -year-old $15 \mathrm{keV}$ measurements of Amaldi et al. [6] to around $0.9 \mathrm{eV}$ in the $20 \mathrm{keV}$ multichannel spectrometer of the Flinders group [7,8] and around $1.5 \mathrm{eV}$ for the new ANU $50 \mathrm{keV}$ multichannel spectrometer [9]. Similarly the momentum resolution has improved from around 1 au in the $9 \mathrm{keV}$ measurements of Camilloni et al. [10], to around 0.1 au in the higher energy Flinders and ANU spectrometers. At the same time the coincidence count rate has improved by several orders of magnitude. The $25 \mathrm{keV}$ measurements on graphite of Gao et al. in 1988 [11] with an energy resolution $\Delta E=8.6 \mathrm{eV}$ (fwhm) and momentum resolution of around $0.3 \mathrm{au}$ had a maximum coincidence count rate of 1 per minute. This compares with the results of Vos et al. [12] on a similar sample of graphite which had coincidence count rates of around 50 per minute with an energy resolution of $1 \mathrm{eV}$ and momentum resolution of around $0.1 \mathrm{au}$. These advances have been made possible by multichannel detection methods, which are employed by the Flinders and ANU spectrometers. These spectrometers are somewhat complementary and their main features and performances will be compared in the following sections.

\section{Kinematics}

Both the Flinders and ANU spectrometers employ non-coplanar kinematics, that is for each polar angle setting at or near the Bethe ridge, the emitted electrons are detected over a range of azimuthal angles out of the scattering plane. In the Flinders spectrometer the arrangement is asymmetric with $\theta_{\mathrm{f}}=14^{\circ}$ and $\theta_{\mathrm{s}}=75.6^{\circ}, E_{0}=20 \mathrm{keV}+\epsilon, \bar{E}_{\mathrm{f}}=18.8$ $\mathrm{keV}$ and $\bar{E}_{\mathrm{s}}=1.2 \mathrm{keV}, K \sim 9 \mathrm{au}$, and $\phi_{\mathrm{f}}$ and $\phi_{\mathrm{s}}$ being varied. The ANU spectrometer employs symmetric kinematics with $\theta_{\mathrm{f}}=\theta_{\mathrm{s}}=44.3^{\circ}, \phi_{\mathrm{f}}$ and $\phi_{\mathrm{s}}$ being varied, and $E_{0}=50 \mathrm{keV}+\epsilon, \bar{E}_{\mathrm{f}}=\bar{E}_{\mathrm{s}}=25 \mathrm{keV}$, with $K \sim 43$ au. The two arrangements are shown in Fig. 1. It should be noted that $\theta_{\mathrm{f}}+\theta_{\mathrm{s}}$, which is chosen to ensure that for $\phi_{\mathrm{f}}-\phi_{\mathrm{s}}=\pi$ the geometry corresponds to that of a collision of the incident electron with a free electron at rest $(q=0, \epsilon=0)$, is in both cases slightly less than $90^{\circ}$ due to relativistic effects.

Through the use of two-dimensional position sensitive detectors placed at the exit of the two electrostatic electron analysers, data is simultaneously acquired over a range of $\phi_{\mathrm{f}}, \phi_{\mathrm{s}}$ and $E_{\mathrm{f}}, E_{\mathrm{s}}[7,9]$.

In both cases under normal settings the measurements give a slice of the spectral momentum density through zero momentum (a $\Gamma$ point) along the $y$ direction. In the Flinders apparatus non-zero values of $q_{x}$ and $q_{z}$ can be chosen by rotating the slow electron (torroidal) analyser about $\theta_{\mathrm{s}}=75.6^{\circ}$, whereas in the ANU apparatus $\theta_{\mathrm{s}}$ and $\theta_{\mathrm{f}}$ can be varied independently by up to $\pm 1^{\circ}$ from $44.3^{\circ}$ by means of a set of double deflector plates along each outgoing trajectory. If both angles are set at $44.3^{\circ}+\delta$ one can measure the energy momentum density in the $y$ direction with a constant momentum component along the $z$-direction. If the voltages to the deflectors are applied asymmetrically, so that one detector measures electrons scattered over $44.3^{\circ}+\delta$ and the
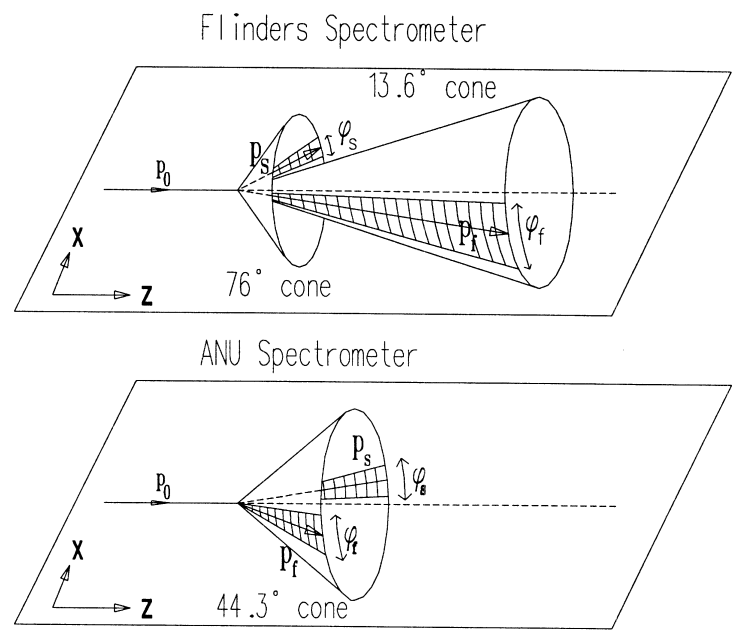

Fig. 1. The non-coplanar kinematics of (a) the Flinders [7] and (b) ANU [9] EMS spectrometers. The incident direction is the $z$ direction and the $x$-direction defines the mean scattering plane. 
other $44.3^{\circ}-\delta$, one measures the $y$-component of the energy-momentum density with a constant component in the $x$-direction (ie. in the mean horizontal scattering plane). This is important for anisotropic samples such as single crystals.

\section{Multiple scattering effects}

High incident energies and outgoing energies are required for solid targets for a number of reasons. Firstly they ensure that the momentum transfer is large and that the plane wave approximation (Eq. (7)) is valid. In addition, and most importantly, they increase the elastic and inelastic mean-free paths of the electrons and thus minimise the deterioration of the data due to multiple scattering events, which are present even with the use of extremely thin selfsupporting targets [13-16].

The degree of multiple scattering is governed by the ratio $t_{j} / \lambda\left(E_{j}\right)$, where $t_{j}$ is the path length in the sample for electron $j=0, \mathrm{~s}, \mathrm{f}$ and $\lambda$ is its mean free path. $t_{j}$ is related to the thickness $T$ of the foil sample and the angle $\Theta_{j}$ the electron $j$ makes relative to sample perpendicular [16]. There is both an elastic and inelastic mean free paths to consider.

Inelastic scattering of a high energy electron in a solid is dominated by plasmon excitation of energy $\Omega_{\mathrm{p}}$. This involves energy loss by the electron and hence the observed binding energy $\epsilon_{\mathrm{obs}}$ is shifted to larger values by the amount $\Omega_{\mathrm{p}}$. If the electron suffers two inelastic collisions or two of the electrons suffer an inelastic collision the energy shift will be $2 \Omega_{\mathrm{p}}$. Since the plasmon energy spread can be quite broad and more than one type of plasmon can contribute, this leads to a tail to the measured intensity reflecting that density shifted in energy and broadened by the widths of the plasmons. The inelastic mean-free path $\lambda_{j}^{i}\left(E_{j}\right)$ depends on the plasmon energy (i.e. density of target electrons) and is at these energies approximately proportional to $E_{j}$. Typically for $10 \mathrm{keV}$ electrons $\lambda^{i}$ is of the order of $10 \mathrm{~nm}$. The decrease of the inelastic scattering cross section and increase of the mean-free path with increasing energy is graphically shown in Fig. 7 of Section 6, where the relative performance of the two spectrometers is discussed.

Elastic scattering on the other hand does not change $\epsilon$ but does change the observed momentum by

$\Delta \mathbf{q}=\mathbf{q}_{\mathrm{obs}}-\mathbf{q}=\Delta \mathbf{k}_{0}-\Delta \mathbf{k}_{\mathrm{f}}-\Delta \mathbf{k}_{\mathrm{s}}$,

with $\Delta \mathbf{k}$ the change of momentum of the incoming and outgoing electrons due to elastic scattering. Since $\mathbf{q}_{\text {obs }}$ is always directed along the $y$-axis, it is clear from Eq. (11) that electrons with $\mathbf{q}$ directed away from the $y$-axis can contribute to the measured events. Thus, target electron with different momenta (and corresponding binding energy) contribute to the intensity at $\mathbf{q}_{\text {obs }}$ [16]. At high energies the elastic scattering can be well described by the Born approximation, and the total elastic mean-free path is $\left(N \sigma_{\mathrm{e}}(E)\right)^{-1}$ where $N$ is the number of atoms per unit volume in the solid and $\sigma_{\mathrm{e}}$ is the total atomic elastic cross section. The momentum change $\Delta \mathbf{q}$ can be estimated from the differential elastic cross section.

These multiple scattering events are still coincident in time, and are part of the coincidence count rate. However, they obscure the system's spectral momentum density and are thus detrimental to the measurement.

In the Flinders asymmetric spectrometer the dominant contribution to multiple scattering effects are due to the slow $1.2 \mathrm{keV}$ electron. This essentially limits the effective target thickness to $2-3 \mathrm{~nm}$ at the exit surface of the foil $[8,17]$. Events taking place deeper within the target would result in the emitted slow electron exciting several plasmons shifting the coincidence event to much higher binding energies. By the same token elastic scattering is more severe for the deeper events.

The surface sensitivity of the Flinders spectrometer can be further enhanced by ensuring that the slow electrons emerge at a large angle $\Theta_{\mathrm{s}}$ relative to the sample normal, increasing the path length $t_{\mathrm{s}}$ for an $(\mathrm{e}, 2 \mathrm{e})$ event taking place at a given distance from the exit surface. Canney et al. [18] used this feature to investigate the spectral density function of an aluminium surface undergoing oxidation, and Vos et al. [19] to demonstrate adsorbate wave function mapping by EMS.

Although the (e,2e) cross section goes as $K^{-4}$, the 'true' signal from the high-energy non-coplanar symmetric ANU spectrometer $(K \sim 43 \mathrm{au})$ is very similar to that obtained with the Flinders spectrome- 
ter $(K \sim 9$ au $)$ in part due to the very significant increase in escape depth of $25 \mathrm{keV}$ electrons compared to $1.2 \mathrm{keV}$ electrons. Most importantly multiple scattering effects are much reduced in the highenergy spectrometer.

Multiple scattering contributions to the data can be treated in two ways. The data can be deconvoluted using an algorithm such as that developed by Jones and Ritter [13]. This works best for the inelastic contributions. Alternatively one can add calculated multiple scattering contributions to a calculated spectral momentum density function, using for instance the Monte-Carlo method of Vos and Bothema [16], and compare this with the raw data.

\section{Target preparation}

For transmission EMS measurements the requirements for target sample preparation are quite severe. The target should be a very thin (10-20 nm) freestanding membrane with a diameter of the order of $0.3 \mathrm{~mm}$. The composition needs to be well known and for experiments in the asymmetric geometry it needs to have a clean exit surface.

Details of typical sample preparation are given by Fang et al. [20]. The preparation and characterisation facilities are shown schematically in Fig. 2. Samples are first thinned by cleaving and/or electrochemical and chemical etching to a preliminary stage. Alternatively films such as those of various forms of amorphous carbon can be simply prepared on a surface which dissolves in solution (e.g. surfactant or salt in water) and the floating films can then be lifted off the surface by a suitable mount. These samples can then be further thinned if desired by either reactive ion (plasma) etching or etching by the ion beam facility, (b) and (c) in Fig. 2. A laser interferometer (d) can be used for monitoring the thickness during the ion etching.

The target preparation and characterisation facility consists of two vacuum chambers connected in series with the main collision chamber. The chamber furthest from the collision chamber has a dual function. It serves as a chamber where reactive etch gases can be used and where the etch gas pressure can be maintained in the Torr range. The intermediate buffer chamber (low $10^{-10}$ Torr range) has

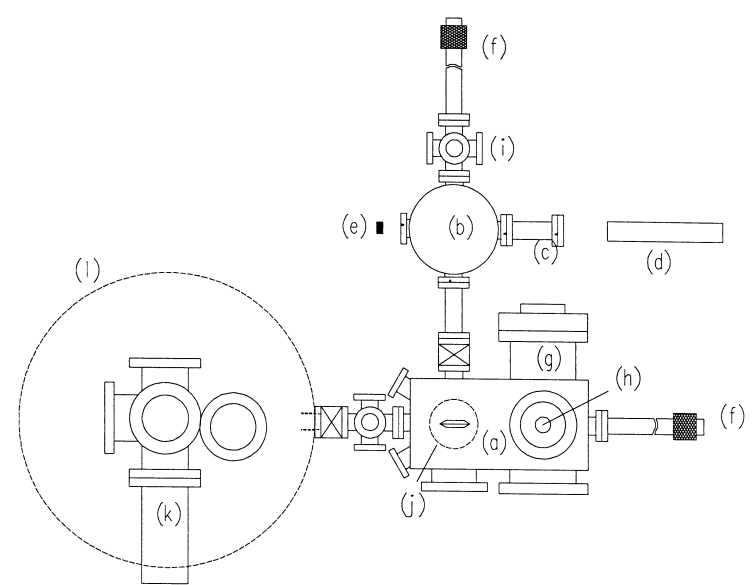

Fig. 2. Plan of the target preparation facilities consisting of UHV preparation chamber (a), (reactive) ion etching chamber (b), ion etching gun (c), laser (d), photon detector (e), transfer arms (f), Auger system for surface analysis (g), sample manipulator and annealing facility (h), load lock and optical microscope for viewing sample (i), evaporator (j), transmission diffractometer (k), and vacuum tank for main spectrometer (1).

an annealing stage and an Auger system for characterising the surface. Targets can also be prepared in this chamber by evaporation onto one surface of a thin free-standing film (usually carbon). Argon ion sputtering can also take place in this chamber. Ultra high vacuum (UHV) conditions are maintained in the preparation chambers, the target samples being inserted using a load-lock system. Similarly, it is possible to transfer the sample rapidly from one chamber to another under UHV conditions.

A transmission electron diffraction facility, mounted on top of the EMS spectrometer can be used to further characterise the sample. The target is mounted on a manipulator in the main spectrometer chamber. As well as movement in the $x, y$ and $z$ directions, this manipulator allows rotation about the vertical ( $y$-axis) and the surface normal. The sample can be aligned along a crystal direction using the diffraction set up, and then transferred to the measurement stage retaining this alignment. Thus, momentum densities can be determined along chosen crystallographic directions.

With these facilities a range of high quality target can be produced. Samples already successfully fabricated include graphite and silicon single crystals, free standing films of copper, aluminium oxide, 
silicon and silicon oxide. For the exit surface sensitive Flinders spectrometer, considerable number of amorphous or polycrystalline targets have been made by evaporating sample material onto a thin amorphous carbon substrate. Samples studied in this way include aluminium, fullerene, silicon, germanium and copper. A $3 \mathrm{~nm}$ overlayer of the material is generally sufficient to attenuate the signal from the carbon backing by several orders of magnitude. In some cases such as copper, where 'islands' are formed on evaporation onto the backing, the characteristic carbon energy-momentum density traces underlie the sample data. However, since the carbon energy-momentum densities have been accurately measured, they can be subtracted from the data.

\section{Energy and momentum resolution}

The observed energy resolution in an EMS measurement is determined by

$\Delta E_{\epsilon}=\left(\Delta E_{0}^{2}+\Delta E_{\mathrm{f}}^{2}+\Delta E_{\mathrm{s}}^{2}\right)^{1 / 2}$,

where it is assumed that all uncertainties in energy have essentially a Gaussian shape and are independent of each other. For thermionic emitters $\Delta E_{0}$ is determined by the thermal spread but space charge effects can broaden the incident beam energy considerably at high currents [1]. This can be overcome by the use of a photo-electron source or more conventionally by the use of a monochromator $[1,8]$. $\Delta E_{\mathrm{f}}$ and $\Delta E_{\mathrm{s}}$ are determined by the analyser resolution and the accuracy of the position decoding.

It is essential to ensure that the voltages determining the different electron energies are all stable over the long term and free of ripples. Commercially available power supplies generally have at best longterm stabilities of about $50 \mathrm{ppm}$, which corresponds to $1 \mathrm{~V}$ at $20 \mathrm{kV}$. It should be noted, however, that new high voltage commercial supplies have become available with stated stabilities of $2.5 \mathrm{ppm}$. Asymmetric kinematics simplifies the problem considerably because $E_{0} \sim E_{\mathrm{f}}$ and $E_{\mathrm{s}}$ is quite low. This means a single high-quality power supply can be used to define the difference in energy $E_{0}-E_{\mathrm{f}}$, although the absolute values of $E_{0}$ and $E_{\mathrm{f}}$, determined essentially by a common high voltage supply, need not be maintained to the same high degree of stability. By using another high-quality supply to define $E_{\mathrm{s}}$ this leads to a stable determination of $\epsilon$. The arrangement used by Storer et al. [7] is shown in Fig. 3.

The layout of the ANU spectrometer is shown schematically as a horizontal cut through the apparatus in Fig. 4. The target specimen is in a large hemisphere which is kept at $+25 \mathrm{kV}$ with respect to ground. The electron gun cathode is kept at $-25 \mathrm{kV}$ plus an additional negative offset voltage to allow for finite binding energies. Thus, inside the floating target region the incident electrons have nominal energy $E_{0}$ of $50 \mathrm{keV}$. Two narrow slits extending over $\pm 6.5^{\circ}$ of azimuthal range are mounted vertically near the exit in the high voltage hemisphere at $\theta_{\mathrm{f}}=\theta_{\mathrm{s}}=44.3^{\circ}$. The two $25 \mathrm{keV}$ emerging electrons are decelerated on leaving the high voltage hemisphere and analysed for energy and azimuthal angle $\phi$ after passing through a hemispherical analyser. A

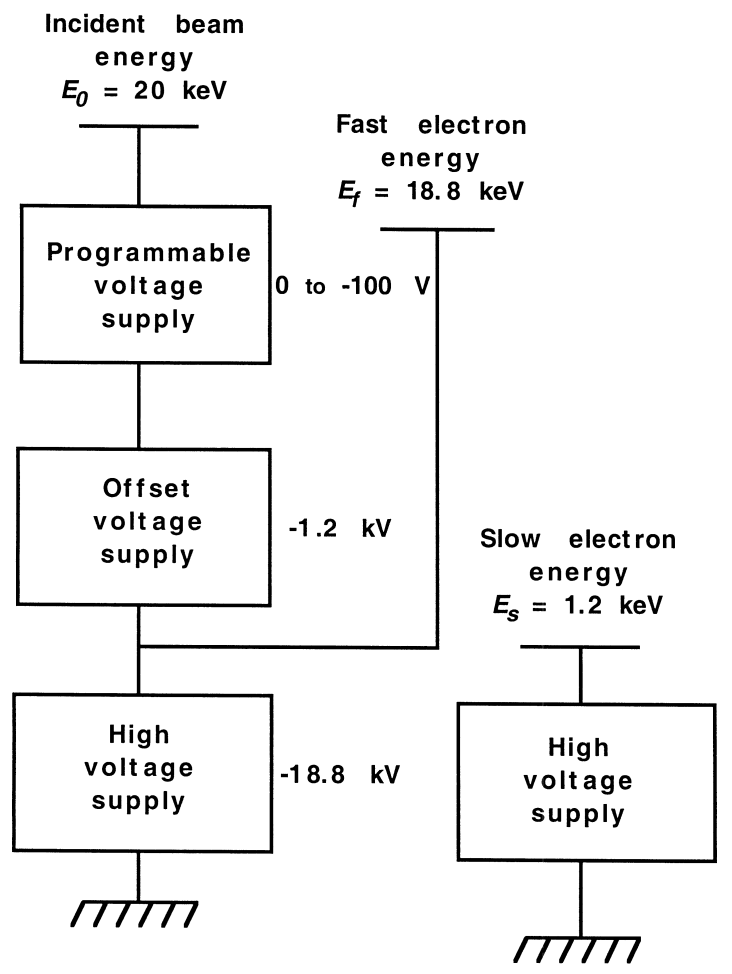

Fig. 3. The configuration of high voltage power supplies used by Storer et al. [7] to define incident and emitted electron beam energies. 


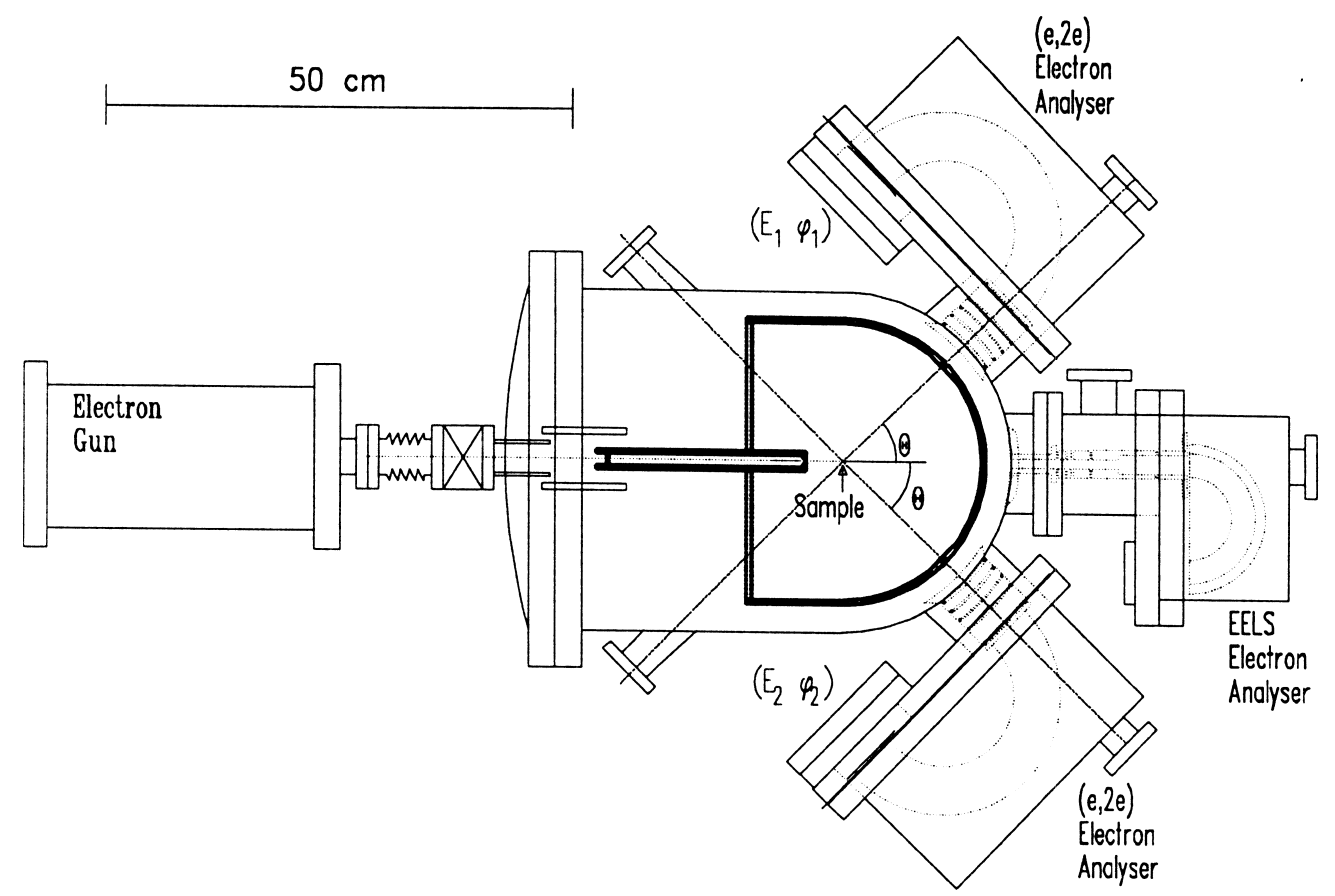

Fig. 4. Horizontal $(x-z$ plane) cut through the ANU high-energy EMS spectrometer of Vos et al. [9]. The positive high-voltage area enclosing the collision region is indicated by the thick line. The analysers and electron optics are indicated as dotted lines.

double pair of deflector plates placed in the hemisphere between the target and exit slits can be used to change the azimuthal angles $\theta$ by up to $\pm 1^{\circ}$ [9].

The high-voltage layout for this spectrometer is similar to that used by Ritter [21] and is shown schematically in Fig. 5. One advantage of this system is that the analysers and detectors are close to ground potential, facilitating coupling of the detector signals to the electronics, and allowing the use of separate high quality, relatively low-voltage supplies for the analysers. The arrangement also helps in meeting the power supply stability requirements. For the main $\pm 25 \mathrm{keV}$ high voltage supplies it is only necessary to ensure that $V_{\text {main }}^{-}=-V_{\text {main }}^{+}$, since except for small offset voltages, which can be accurately maintained and determined, $E_{0}=-V_{\text {main }}^{-}+V_{\text {main }}^{+}$and $E_{\mathrm{f}}=E_{\mathrm{s}}=$ $V_{\text {main }}^{+}$. Drifts and ripple may occur as long as they do not affect the equality $V_{\text {main }}^{-}+V_{\text {main }}^{+}=0$. The long term drift compensation is achieved by measuring the midpoint potential of an accurate and highly stable temperature controlled divider chain that connects the output of both power supplies. The ripple in $V_{\text {main }}^{-}+V_{\text {main }}^{+}$is measured by a capacitive divider. A small correction voltage is then applied at the base of one of the main supplies. After applying the feedback $V_{\text {main }}^{-}+V_{\text {main }}^{+}$is constant within 100 $\mathrm{mV}$. Further, all of the electronics floating at \pm 25 $\mathrm{keV}$ is battery powered to eliminate AC ripple, which can arise from the use of isolation transformers. The batteries need recharging after about 6 days.

The momentum resolution of an EMS spectrometer is controlled largely by the angular resolution of the incident and emitted electron beams. For multiparameter detectors, where a range of emitted energies is detected simultaneously, the contribution of the energy spread to the momentum spread may be significant. Thus, a $20 \mathrm{eV}$ range in energy corresponds to a range of momenta of 0.08 au for a 1.2 $\mathrm{keV}$ electron. For a $25 \mathrm{keV}$ electron, an energy range of $100 \mathrm{eV}$ also corresponds to a momentum range of around 0.08 au. However, since the energy of each detected electron is recorded online in the multiparameter data acquisition systems of Storer et al. [7] and Vos et al. [9], the momentum conservation Eq. (2) can be accurately calculated for each event in the software. 


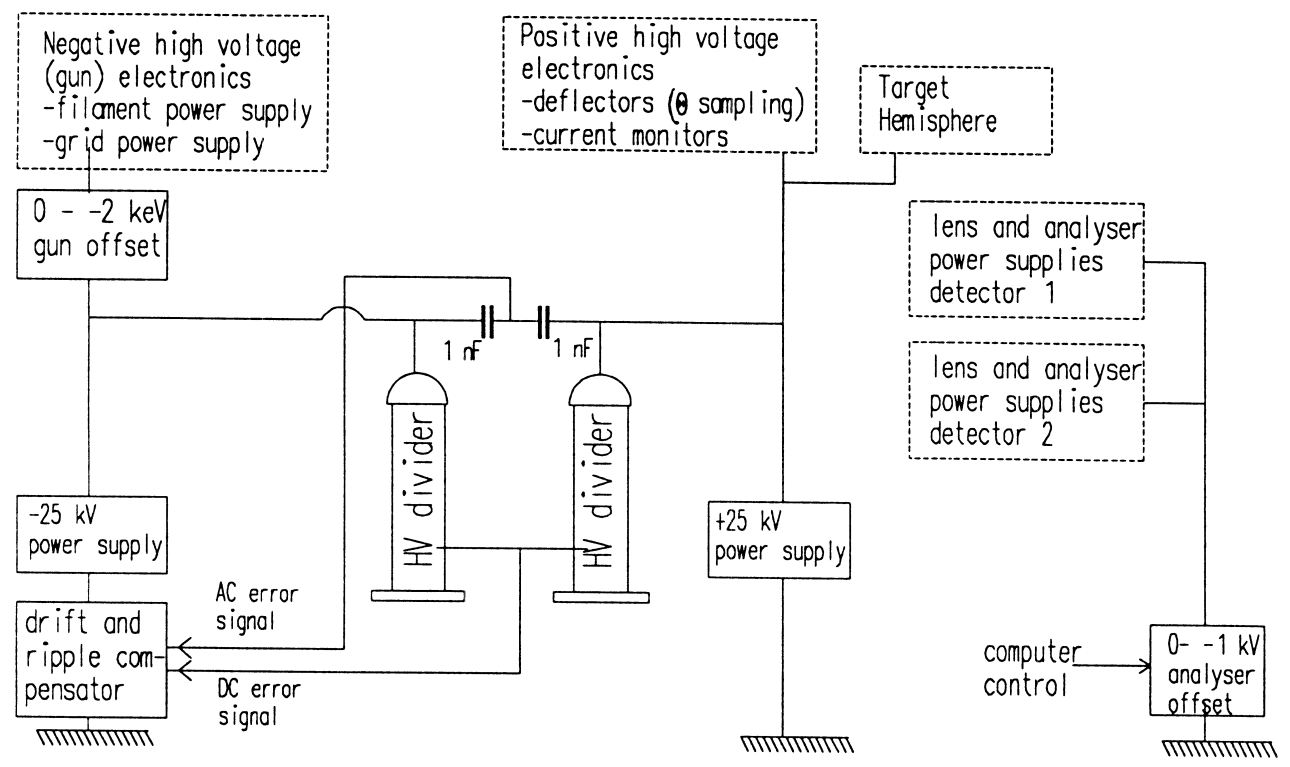

Fig. 5. An overview of the ANU high-voltage configuration and its stabilisation system [9].

The angular divergence of the incident beam can generally be kept very small. Similarly the spot size at the target can be kept to below $0.25 \mathrm{~mm}$ diameter. This spot size and the slit widths before the decelerating stage determine the angular divergence in $\theta$ of the emerging beams. The full width of polar angular spread is thus given by

$\Delta \theta=\frac{w_{\mathrm{s}}+w_{\mathrm{t}}}{d}$,

where $d$ is the distance from the target to slit and $w_{\mathrm{t}}$ the beamsize at the target and $w_{\mathrm{s}}$ the slit width. For the ANU spectrometer this leads to a full width in outgoing momentum spread of around 0.1 au for $w_{\mathrm{s}}=0.2 \mathrm{~mm}$ and $w_{\mathrm{t}}=0.1 \mathrm{~mm}$. The full width half maximum is much less than this, leading to very small uncertainties in the $x$ and $z$ components of momentum.

Calibration of the angular and energy sensitivity of each analyser is carried out using a set of apertures of well defined diameter at known azimuthal angles and taking elastic scattering measurements with a thin ( $\sim 5 \mathrm{~nm}$ thick) amorphous carbon target foil, changing the energy by well defined steps. Fig. 6 shows a typical energy sweep for $25 \mathrm{keV}$ electrons across the channel plates and two-dimensional position-sensitive detector mounted at the exit of one of the hemispherical analysers in the ANU spectrometer. The left-right direction corresponds to changes in energy and the top-bottom direction to azimuthal angle. The elastic peaks are located on circular segments which in the figure are separated by $7 \mathrm{eV}$ for a mean pass energy through the analyser of $400 \mathrm{eV}$. Data such as these are used to calibrate each detector. The centroid for the position of each peak of known energy and angle is used as input to a fitting program [22], which can then be used to determine the angle and energy of each detected electron.

\section{Spectrometer comparison}

It is interesting to compare the performance of both spectrometers with typical target specimens. By tuning the incident energy to the energy of the analysers one obtains single electron scattering spectra for elastic and inelastic scattering. Fig. 7 shows the results for the Flinders spectrometer using an amorphous carbon sample $10 \mathrm{~nm}$ in thickness. Whereas inelastic processes are quite small at 18.8 $\mathrm{keV}$ (and even smaller at 25 and $50 \mathrm{keV}$ ) relative to the elastically scattered peak, they are very large at $1.2 \mathrm{keV}$. In the $18.8 \mathrm{keV}$ data the excitation of single 

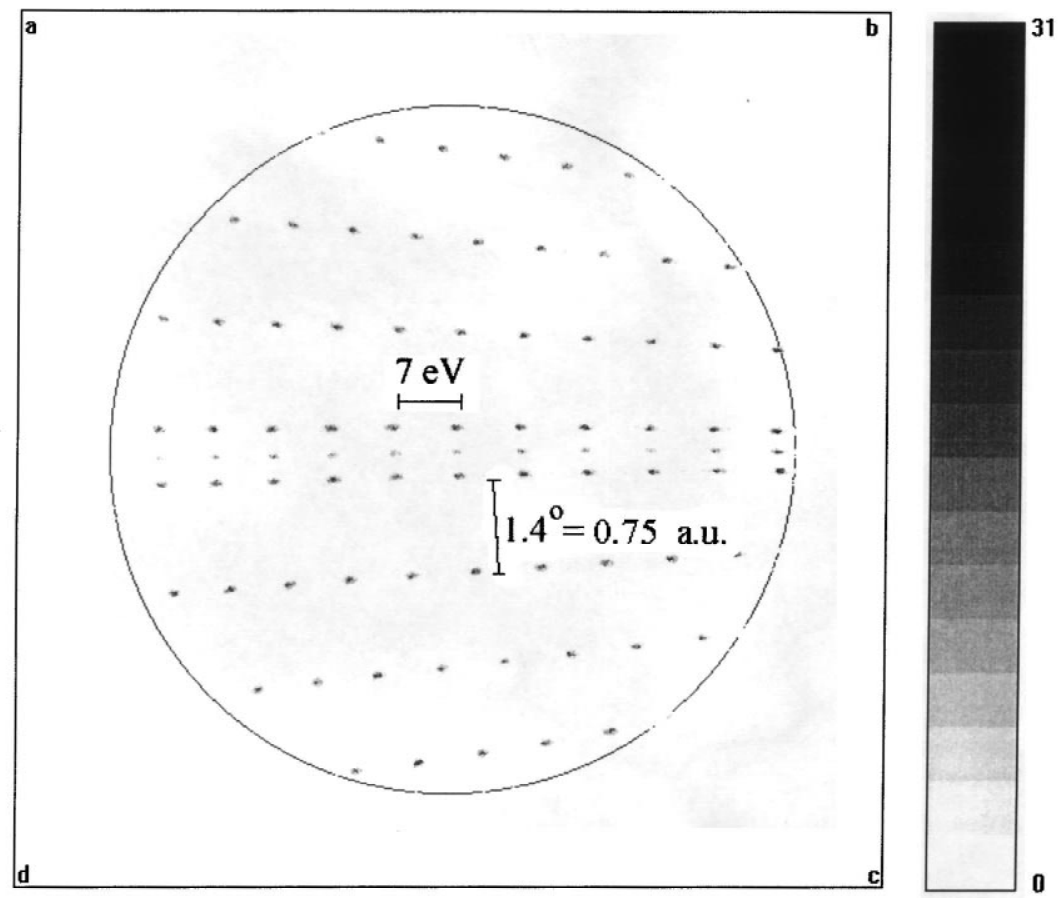

Fig. 6. Position sensitive detector output for a calibration of one hemispherical analyser of the ANU spectrometer obtained by elastic scattering of electrons through apertures of $0.1 \mathrm{~mm}$ diameter separated by known azimuthal angles. The incident energy of nominally $25 \mathrm{keV}$ was varied in $7 \mathrm{eV}$ steps.

$\pi$ and $\sigma$ plasmons, at energy losses of around $6 \mathrm{eV}$ and $23 \mathrm{eV}$, respectively, dominates the inelastic spectrum. Although the $\pi$ plasmon peak is clearly visible at $1.2 \mathrm{keV}$, the inelastic spectrum is dominated by multiple plasmon creation. The short inelastic mean-free path for $1.2 \mathrm{keV}$ electrons is responsible for the surface sensitivity of the Flinders spectrometer.

Clearly inelastically scattered electrons will lead to coincident detection of electrons with observed binding energies significantly below the actual valence band energies. Indeed the energy loss can be so large as to lead to a coincidence background even at a binding energy corresponding to the core $1 \mathrm{~s}$ band of carbon at $\epsilon_{1 \mathrm{~s}} \simeq 288 \mathrm{eV}$. This coincidence background with a carbon target was studied in detail by Vos et al. [17] for the Flinders spectrometer. They found a continuous background due to multiple scattering events, which decreased with binding energy but was still quite significant at the core binding energy. The much higher energies used in the ANU spectrometer result in much reduced multiple-scattering and essentially no coincident background at the core binding energy. This can be seen in Fig. 8 which compares the core binding energy spectra obtained with both spectrometers.

Fig. 9 shows the spectral-momentum density obtained from similar samples of highly-oriented pyrolitic graphite (HOPG) with the Flinders and ANU spectrometers. Here $q_{x}=q_{z}=0$ and the intensity is plotted in uniform grey scale as a function of $q_{y}$ and $\epsilon$. The energies are relative to the vacuum level, the Fermi level is approximately $4 \mathrm{eV}$ below the vacuum level.

The difference between HOPG and single crystal natural graphite is that the order perpendicular to the $c$-axis (i.e. in the graphite plane) is lost in HOPG. The randomly oriented crystallites are of appreciable size, so essentially all atoms are still in a perfect crystal environment. The sample was mounted to align the $c$-axis with the $z$-direction, ie. the graphite planes were perpendicular to the incident direction. 

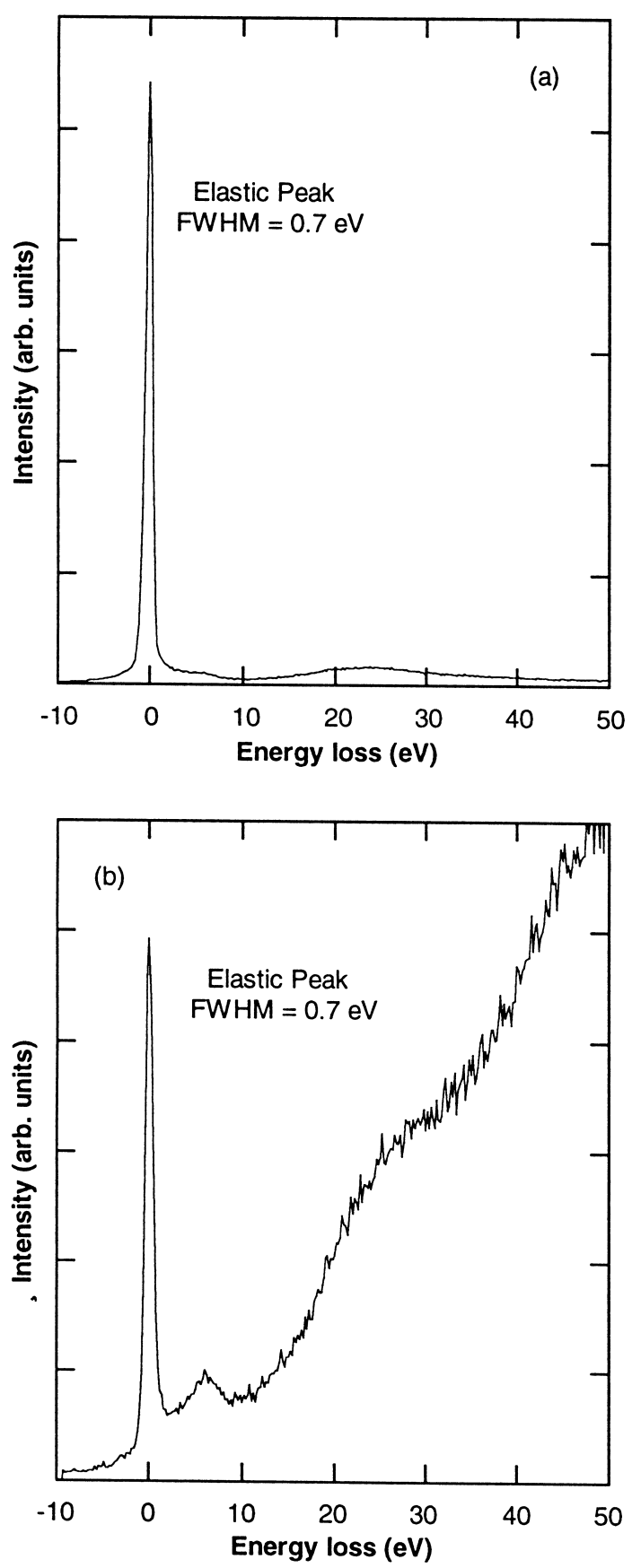

Fig. 7. Electron energy loss spectra from a $10 \mathrm{~nm}$ thick amorphous carbon foil for (a) $18.8 \mathrm{keV}$ electrons scattered by $\theta_{\mathrm{f}}=14^{\circ}$ and (b) $1.2 \mathrm{keV}$ electrons scattered by $\theta_{\mathrm{s}}=75.6^{\circ}[8]$.
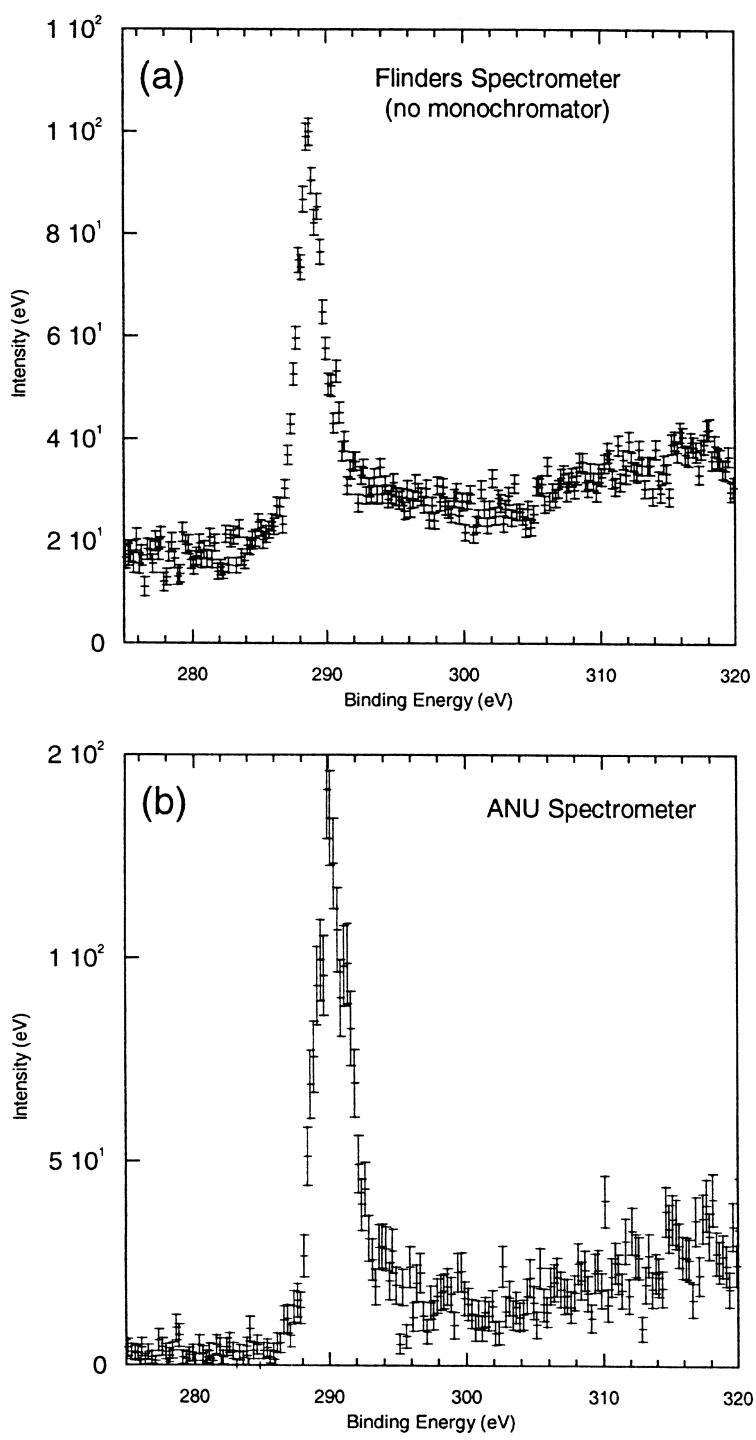

Fig. 8. Core binding energy spectra for similar amorphous carbon foils obtained with (a) the Flinders asymmetric spectrometer [8] and (b) the high energy ANU spectrometer.

The $\pi$ band has a node in the $x-y$ plane, so the density of $\pi$ electrons should be zero in the $q_{z}=0$ plane. Indeed the observed $\pi$-electron density is very small in Fig. 9, some small $\pi$ intensity being expected due to the finite momentum resolution and elastic scattering.

The dominant feature is the dispersive $\sigma$ band. The lack of sharp features near the top of the band is due to averaging out over $\Gamma-M, \Gamma-K$ and inter- 


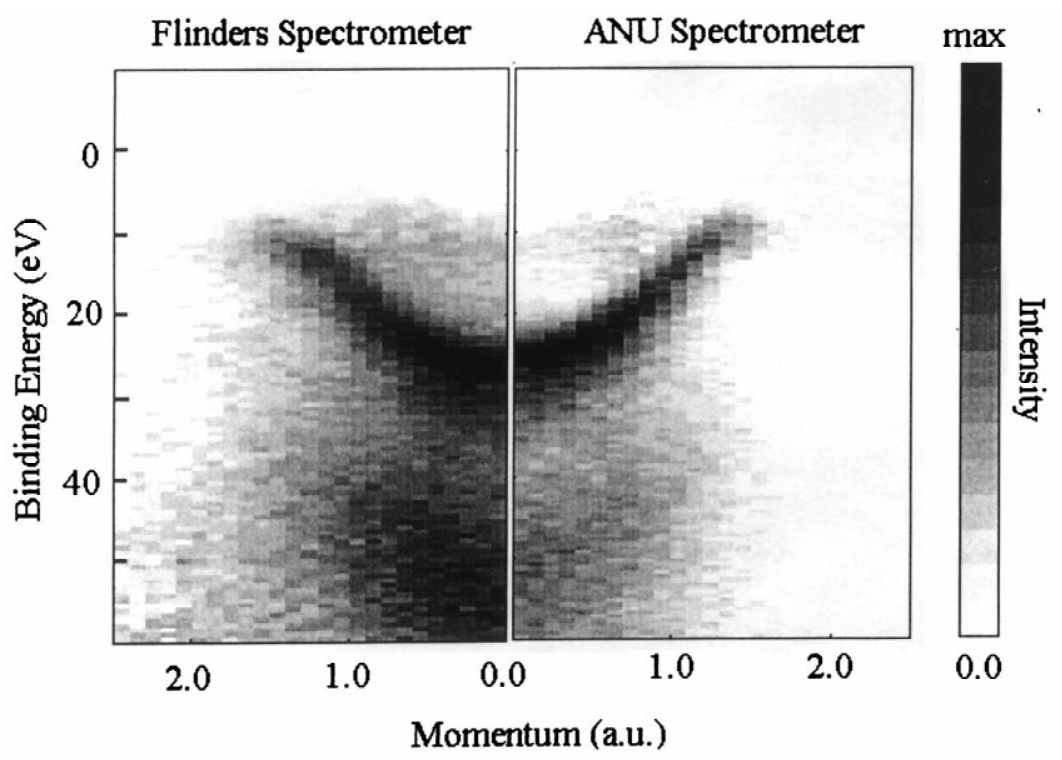

Fig. 9. The spectral-momentum density of the valence bands of highly-oriented pyrolite graphite (HOPG) for $q_{x}=q_{z}=0$. Left panel is the Flinders data [24] and right panel is ANU data.

mediate directions in the graphite plane. The width of the band, particularly near the bottom, i.e. $\Gamma$ point, is significantly large than the experimental energy resolution. This width is due to electron correlation effects and the resulting short quasiparticle lifetime. A significant fraction of the observed intensity below the $\sigma$ band is due to electron correlations, i.e. satellite structure. Multiple-scattering effects due to plasmon excitation and elastic scattering are present. They are significantly smaller in the higher energy data (right panel) than in the earlier measurements with the asymmetric spectrometer [23,24].

The significance of multiple scattering can be more clearly displayed by taking cuts in Fig. 9 along either the momentum or energy axes. Fig. 10 plots the observed binding energy spectra at $q=0$ $\left(-0.1 \leq q_{y} \leq 0.1\right)$ for the two different spectrometers, normalised to the same peak maximum for the $\sigma$ band. Although some of the structures above the $\sigma$-peak at $25 \mathrm{eV}$ below the vacuum level is real structure and due to many-body effects, contributions due to plasmon excitation can clearly be seen. They are particularly strong for the measurements using the asymmetric spectrometer.

As discussed earlier, elastic scattering contributions to multiple-scattering effects can provide a

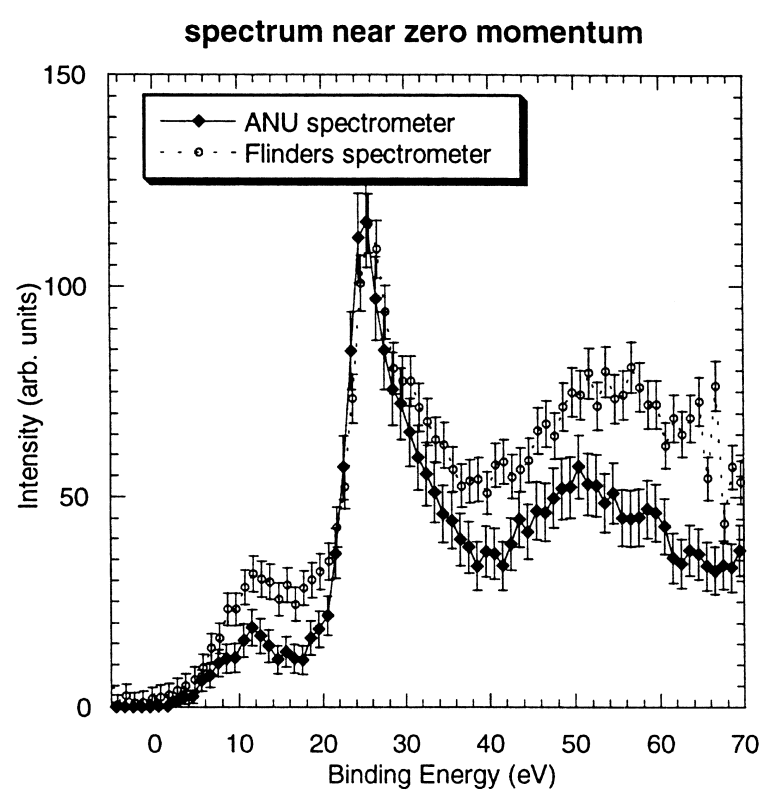

Fig. 10. Energy spectra near zero momentum from Fig. 9 for similar specimens of HOPG with the ANU high energy spectrometer (solid diamonds) and the asymmetric Flinders spectrometer (open circles). The data have been normalised to the same $\sigma$ peak intensity. 
smeared out background to the 'real' spectral momentum density. Collision (e,2e) events at $(\boldsymbol{\epsilon}, \mathbf{q})$ off the $q_{y}$-axis can be projected to a point $q_{y}$ on that axis by elastic scattering with $\Sigma_{j} \Delta \mathbf{k}_{j}=\mathbf{q}-q_{y}$ (Eq. 11). The most obvious effect on the data is to fill in the region between the dispersing arms of the $\sigma$ band. This can be seen in Fig. 10 as extra intensity at binding energies smaller than those corresponding to the $\sigma$ peak. Some of this extra intensity arises from electrons in the $\pi$ band.

Elastic scattering contributions can also be seen in Fig. 11, which shows a cut in the intensity distribution at a constant binding energy of $13 \mathrm{eV}$ as a function of $p_{y}$. The extra intensity between the two $\sigma$ band peaks and beyond them is due to elastic scattering contributions to multiple scattering. Again these effects are significantly reduced by using higher energies.

The coincidence timing spectra obtained for the two measurements displayed in Fig. 9 are shown in Fig. 12. The spectra have been corrected for variations in transit time through the analysers. The new

\section{Momentum density at $13 \mathrm{eV}$ binding energy}

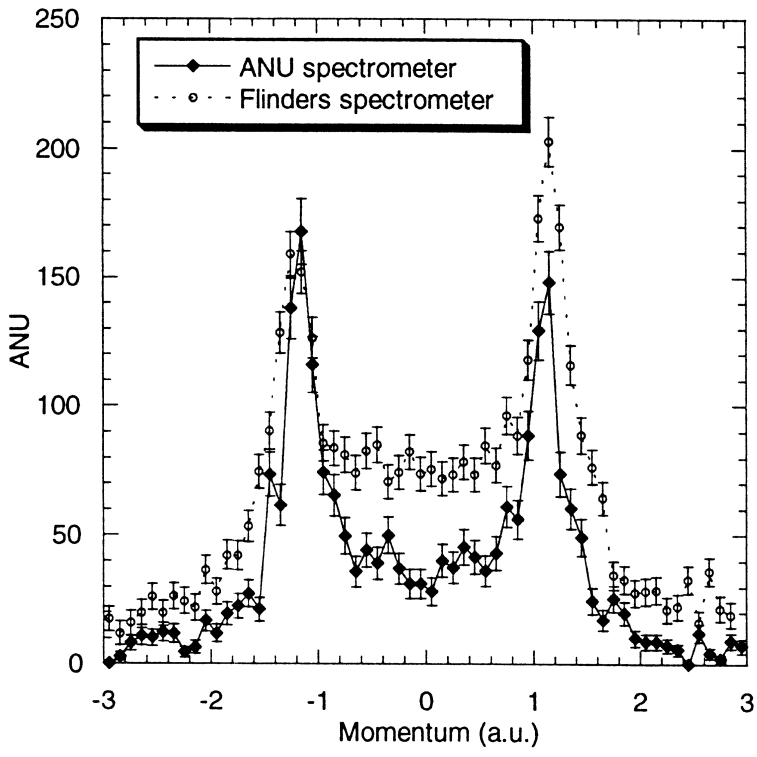

Fig. 11. Momentum density near $\epsilon=13 \mathrm{eV}$ for the HOPG specimens (Fig. 9) obtained with the ANU spectrometer (solid diamonds) and Flinders spectrometer (open circles). Normalisation as in Fig. 10.

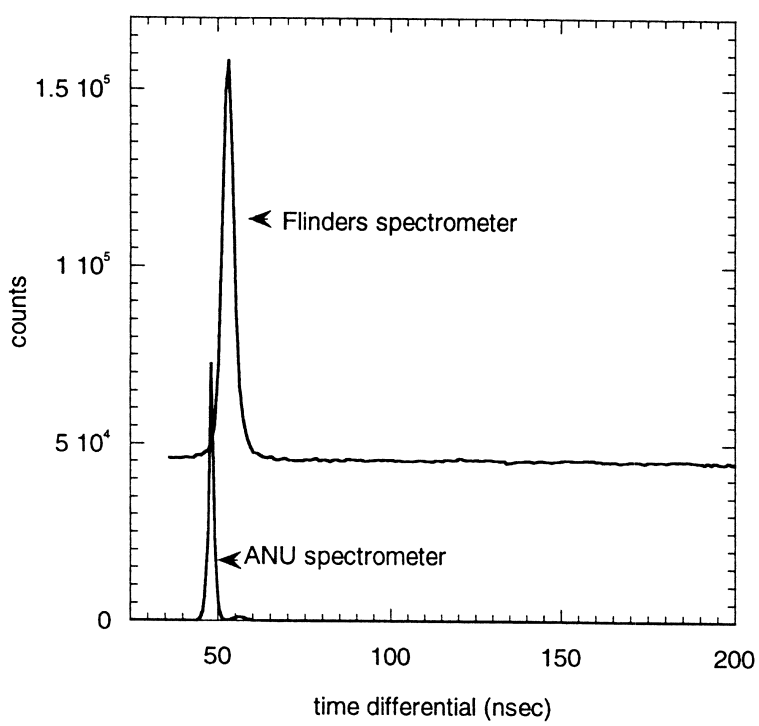

Fig. 12. Timing spectra, corrected for transit times in the analysers, for similar HOPG samples (Fig. 9) obtained with the two spectrometers.

ANU high energy spectrometer has almost no random background underlying the coincident peak.

The valence band (energy integrated) momentum densities obtained with the two spectrometers for similar annealed polycrystalline carbon specimens is compared with LMTO calculated densities [25] in Fig. 13. The Flinders data [25] (open circles) show enhanced high-momentum components due to multiple scattering. With the ANU high-energy spectrometer the effects of multiple scattering are much reduced.

Finally it is interesting to look at the observed count rates for targets of different thickness with both spectrometers. At Flinders the coincidence count rate is strongly dependent on target thickness. It is maximum for the thinnest target measured ( $\approx 50 \AA$ about $6 \mathrm{~Hz}$ at $50 \mathrm{nA}$ beam current $)$ and decreases strongly with thickness to about $0.2 \mathrm{~Hz}$ at $200 \AA$. Without multiple scattering the coincidence rate would increase linearly with thickness. The strong decrease is thus another indication of the importance of multiple scattering. At the ANU the count rate is constant within a factor of two over this thickness range $(1 \mathrm{~Hz}$ at $1.5 \mu \mathrm{A}$ beam current), 


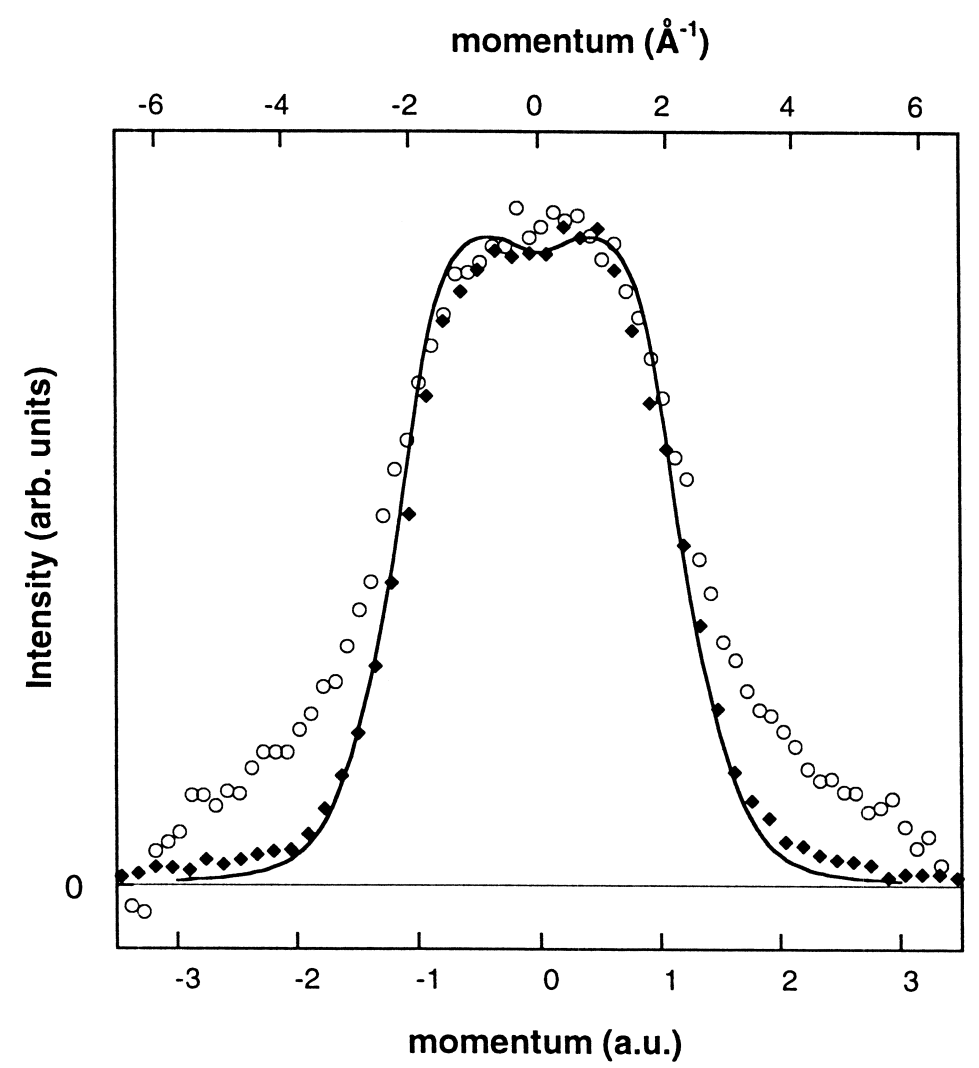

Fig. 13. The valence momentum density (energy integrated to the bottom of the $\sigma$ band) for similar annealed polycrystalline carbon samples obtained with the ANU spectrometer (solid diamonds) with a $35 \AA$ thick sample and the Flinders spectrometer (open circles) with a $60 \AA$ thick sample [25]. The solid curve is the momentum density given by a LMTO calculation [25].

indicating that the increase in thickness is largely compensated by an increase in multiple scattering.

\section{Summary}

The two multiparameter non-coplanar transmission spectrometers at Flinders University and ANU are able to obtain data with good momentum and energy resolution on details of the electronic structure of occupied bands in crystalline, polycrystalline and amorphous specimens. The asymmetric Flinders spectrometer is sensitive to only about $2-3 \mathrm{~nm}$ of the sample on the exit side of the thin target specimen. This has advantages in studying surface effects or in studying samples which can be evaporated on the exit side of a very thin carbon foil. Work along these lines is continuing at Flinders [26,27]. The new high-energy symmetric spectrometer at ANU on the other hand is more bulk sensitive and has the advantage of having much reduced multiple scattering effects. This is particularly important in determining structure due to electron correlation effects.

\section{References}

[1] E. Weigold, I.E. McCarthy, Electron Momentum Spectroscopy, Kluwer Academic/Plenum Press, New York, 1999.

[2] I.E. McCarthy, E. Weigold, Phys. Rep. 27C (1991) 275. 
[3] I.E. McCarthy, E. Weigold, Electron-Atom Collisions, Cambridge University Press, Cambridge, 1995.

[4] S. Dey, I.E. McCarthy, P.J.O. Teubner, E. Weigold, Phys. Rev. Lett. 34 (1975) 782.

[5] W.F. Ford, Phys. Rev. B 133 (1964) 1616.

[6] U. Amaldi Jr., A. Egidi, R. Marconero, G. Pizzella, Rev. Sci. Instrum. 40 (1969) 1001.

[7] P. Storer, R.S. Caprari, S.A.C. Clark, M. Vos, E. Weigold, Rev. Sci. Instrum. 65 (1994) 2214.

[8] S.A. Canney, M.J. Brunger, I.E. McCarthy, P.J. Storer, S. Utteridge, M. Vos, E. Weigold, J. Electron Spectrosc. Relat. Phen. 83 (1997) 65.

[9] M. Vos, G.P. Cornish, E. Weigold, Rev. Sci. Instrum. 71 (2000) 331.

[10] R. Camilloni, A. Giardini Guidoni, R. Tiribelli, G. Stefani, Phys. Rev. Lett. 29 (1972) 618.

[11] C. Gao, A.L. Ritter, J.R. Dennison, N.A.W. Holzwarth, Phys. Rev. B 37 (1988) 3914.

[12] M. Vos, Z. Fang, S.A. Canney, A.S. Kheifets, I.E. McCarthy, E. Weigold, Phys. Rev. B 56 (1997) 963.

[13] R. Jones, A.L. Ritter, J. Electron Spectrosc. Relat. Phen. 40 (1986) 285.

[14] J.R. Dennison, A.L. Ritter, J. Electron Spectrosc. Relat. Phen. 77 (1996) 99.

[15] P. Hayes, M.A. Bennett, J. Flexman, J.F. Williams, Phys. Rev. B 38 (1988) 13371.
[16] M. Vos, M. Bottema, Phys. Rev. B 54 (1996) 5946.

[17] M. Vos, P. Storer, A.S. Kheifets, I.E. McCarthy, E. Weigold, J. Electron Spectrosc. Relat. Phen. 76 (1995) 103.

[18] S.A. Canney, M. Vos, A.S. Kheifets, X. Guo, I.E. McCarthy, E. Weigold, Surface Sci. 382 (1997) 241.

[19] M. Vos, S.A. Canney, P. Storer, I.E. McCarthy, E. Weigold, Surface Sci. 327 (1995) 387.

[20] Z. Fang, X. Guo, S. Utteridge, S.A. Canney, I.E. McCarthy, M. Vos, E. Weigold, Rev. Sci. Instrum. 68 (1997) 4396.

[21] A.L. Ritter, J.R. Dennison, J. Dunn, Rev. Sci. Instrum. 55 (1984) 1280.

[22] R. Caprari, Comput. Phys. 7 (1993) 336.

[23] M. Vos, A.S. Kheifets, E. Weigold, S.A. Canney, F.F. Kurp, J. Electron Spectrosc. Relat. Phen. 87 (1998) 231.

[24] M. Vos, P. Storer, S.A. Canney, A.S. Kheifets, I.E. McCarthy, E. Weigold, Phys. Rev. B 50 (1994) 5653.

[25] F.F. Kurp, M. Vos, Th. Tschentscher, A.S. Kheifets, J.R. Schneider, E. Weigold, F. Bell, Phys. Rev. B 55 (1997) 5440.

[26] S.A. Canney, V.A. Sashin, M.J. Ford, A.S. Kheifets, J. Phys. Condens. Matt. 11 (1999) 7507.

[27] V.A. Sashin, S.A. Canney, M.J. Ford, M.A. Bolorizadeh, D.R. Oliver, A.S. Kheifets, J. Phys. Condens. Matt. 12 (2000) 125. 